\title{
Methodological Difficulties in the Conduct of Neuroepidemiological Studies in Low- and Middle-Income Countries
}

\author{
Nadir Bharucha ${ }^{a, b}$ Peter Odermatt ${ }^{c, d}$ Pierre-Marie Preux ${ }^{\mathrm{e}-\mathrm{g}}$ \\ a Department of Neurology, Bombay Hospital Institute of Medical Sciences, and ${ }^{\text {b}}$ Department of \\ Neuroepidemiology, Medical Research Centre, Bombay Hospital, Mumbai, India; ' Department of Epidemiology and \\ Public Health, Swiss Tropical and Public Health Institute, and dUniversity of Basel, Basel, Switzerland; ${ }^{\mathrm{C} C H U}$ Limoges, \\ Service de l'Information Médicale et de l'Evaluation, Unité Fonctionnelle de Recherche Clinique et de Biostatistique, \\ and fIFR 145 GEIST, Institut d'Epidémiologie Neurologique et de Neurologie Tropicale, EA 3174 NeuroEpidémiologie \\ Tropicale et Comparée, and ${ }^{9}$ Laboratoire de Biostatistique et d'Informatique Médicale, Faculté de Médecine, \\ Université de Limoges, Limoges, France
}

\section{Key Words}

Developing countries · Systematic reviews - Meta-analyses . Burden of epilepsy - Treatment gap

\begin{abstract}
Background: The majority of people with epilepsy (PWE) live in low- and middle-income countries (LMICs). However, they remain largely untreated and the bulk of resources are used to treat patients in the developed world. This disparity constitutes a challenge for neuroepidemiological studies on a global scale. In the past, several studies have focused on diverse populations in disparate countries at various periods of time and for particular purposes. The specificity of different contexts and circumstances makes it difficult to analyse PWE as a group either qualitatively or quantitatively. Such methodological limitations are further complicated by a lack of logistical support. There is a lack of interest in conducting studies, which results in inadequate funding and, in addition, there is the considerable challenge of publishing research reports from LMICs in peer-reviewed international journals. Methods: This paper focuses on methodological problems
\end{abstract}

\section{KARGER}

E-Mail karger@karger.com

www.karger.com/ned related to studies in LMICs and attempts to give the reasons for their limitations using epilepsy as an example. Results: Regional conditions and environmental factors must be given careful consideration in the research design because of the importance of understanding the challenges of living in these environments. There are further limitations to the successful implementation of studies. Existing information on epilepsy is often not readily accessible; there is a lack of census data, and migratory patterns into cities make enumeration and sampling even more challenging. As there is usually no well-developed healthcare system a door-to-door screening process is often the only way to identify those with convulsive epilepsy. The questionnaire and study design should preferably be adapted from standardized protocols, and pretested and validated in local conditions. Conclusions: Systematic reviews and meta-analyses of studies in LMICs can provide data on the burden, risk factors, treatment and outcome of epilepsy only if the primary studies used are properly conducted using uniform and comparable methodology. The use of consistent replicable neuroepidemiological methods in primary studies and systematic reviews enable reduction of the treatment gap and better epilepsy care.

(c) 2013 S. Karger AG, Base

Dr. Nadir Bharucha

Department of Neurology, Bombay Hospital Institute of Medical Sciences Medical Research Centre

12-Marine Lines, Mumbai 400020 (India)

E-Mail nebharucha@gmail.com 


\section{Introduction}

The majority of resources utilized in the treatment of epilepsy worldwide is accounted for by high-income countries. However, the majority of people with epilepsy (PWE) live inlow- and middle-income countries (LMICs), most of them in the tropics. Therefore, most PWE do not receive appropriate treatment, which is known as the treatment gap [1]. Meinardi et al. [2] identified the following factors as being mainly responsible for the treatment gap: inadequate case ascertainment in a population, failure to diagnose epilepsy, and lack of appropriate treatment of epilepsy, which could include medical, surgical or sociocultural intervention. In addition, the treatment gap can also be due to lack of treatment of an underlying cause of epilepsy and failure to continue treatment once started. The latter is often referred to as secondary treatment gap.

Unfortunately there are only a few incidence studies of epilepsy in LMICs, there are several prevalence studies with different study designs, usually identifying convulsive epilepsy in which the aetiology is unknown, hardly any natural history studies, few unbiased case-control studies, and a number of studies mentioning the size of treatment gap but few which analyse it.

There are a few systematic reviews and meta-analyses concerning some of the above issues. Most of them have some or all of the following problems: insufficient scrutiny of the existing literature, methodological differences, differences in case ascertainment, differing definitions of epilepsy and active epilepsy, lack of age-adjustment, use of populations which may not be representative of the general population. Judgment is needed on the part of the person carrying out the meta-analysis about the weighting given to every study included. Weighting requires assessment of methodological quality, i.e. the effort made to minimize bias or systematic error, precision of each study as expressed by the width of the $95 \%$ confidence interval, and the external validity of the study which is the extent to which one may generalize its results [3]. These metaanalyses of incidence studies [4], prevalence studies [5] and treatment gap in epilepsy $[6,7]$ have demonstrated a high degree of unexplained heterogeneity. This is possibly due to the methodological differences and judgments about which studies to include or exclude [8]. Problems with meta-analyses are greatest when there are few primary studies, as with incidence studies, and where there is wide variation in rates $[4,9]$.

Consistently applicable epidemiologic methods are needed in LMICs to generate comparable data on the bur- den of epilepsy, outcome of interventions, risk factors and treatment. Systematic reviews and meta-analyses using such well-executed primary studies would be of use to all those involved in the care of epilepsy.

\section{Regional Difficulties}

There are various difficulties in the use of globally applicable techniques in LMICs. The purpose of this paper is to highlight such fundamental problems and challenges.

LMICs display certain common characteristics. Often, the environment is marked by a specific tropical, equatorial or subtropical climate. There is poor sanitation, which promotes the spread of infections. Environmental toxins are common and people are malnourished. Individual, population-based, cross-sectional and case control studies of active convulsive epilepsy were carried out in five centres in sub-Saharan Africa [10]. In order to reduce heterogeneity of findings, the authors used the same methods and definitions between regions. Interestingly, they found that heterogeneity could be accounted for by markers of birth trauma, exposure to a range of parasites and other factors including malnutrition and ingestion of cassava [10]. The relationship between epilepsy and malnourishment is a complicated one. In order to ascertain which came first, one would need a long-term cohort study of people with malnutrition or with epilepsy [11]. Such studies would be very difficult to conduct in LMICs.

Scarce resources also lead to poor access to care and greater inequality in access. Meta-analysis of prevalent studies of active epilepsy and lifetime epilepsy throughout the world found the prevalence of epilepsy was highest in rural areas of LMIC, lower in urban parts of LMIC and lowest in high-income countries [5]. The authors felt that access to healthcare was an important determinant of these results.

There is inadequate public health and the health systems are also inadequate. There are many specific sociocultural characteristics that may by themselves be risk factors for neurological disease. The population is young, with a low life expectancy compounded by the HIV/AIDS pandemic. There is currently rapid and uncontrolled urban and suburban growth, again creating a mismatch in healthcare provision. Political factors, i.e. unstable governments, multiple levels of decision making and the low priority given to health programs, worsen the situation. In particular, curative and preventive neurological health services are often very weak or lacking altogether. 
A recent study of treatment gap in Kilifi, Kenya, found a treatment gap of $62.4 \%$ which was attributable to a number of factors including traditional beliefs, negative attitudes, chronic epilepsy, focal seizures, learning difficulties, living far from health facilities and having to pay for treatment [12]. It is important for any investigator to assess this 'big picture', not only from the point of view of designing a good study, but also to better place epilepsy in the context of local difficulties.

Carrying out epidemiological research in these settings poses difficulties for a variety of reasons - methodological, logistic, political, economic, ethical and often the low-perceived value of such work [13]. Also, the enormous diversity of such populations makes it difficult to generalize.

\section{What Is Known?}

As a first step, it is essential to ascertain what work has already been accomplished in the area of interest. The primary difficulty is identifying what is known about epilepsy and PWE in the region to be studied.

Obtaining existing information on epilepsy in a new region can be challenging. Published studies are scarce in these areas, and it is often difficult to obtain historical information. Those studies that do exist are frequently difficult to compare because their methodologies are not standardized and sometimes there is duplication [14].

An extensive literature search can provide unexpected historical data. For example, the authors in a study about epilepsy in Lao People's Democratic Republic (Lao PDR) initially found no data in the literature. However, consultations with relevant experts and health authorities revealed that an investigation had been performed on the state of mental illness in Lao PDR by the Ministry of Health years before, and that the report of the survey mentioned a number of data on epilepsy [15]. Therefore, activity reports of ministries and various organizations are more likely to provide results of previous studies than the search in peer-reviewed published literature.

Studies are not necessarily indexed in major international bibliographic databases, and it is necessary to search for national publications or 'grey' literature. Many theses and less formal accounts have described neurological problems in the tropics. There is a virtual library of African neurology on the website of the Institute of Neurological Epidemiology and Tropical Neurology (wwwient.unilim.fr). Another database is the Wan Fang Database of English and Chinese online journals published in mainland China (www.wanfangdata.com).

Methodological Difficulties in the Conduct of Neuroepidemiological Studies in LMICs

\section{Methodology}

\section{Identifying an Area and Population}

Methodological standards are the same everywhere, reflecting the universal need to collect data on which to base a detailed protocol. This process may be difficult because of a lack of accurate demographic data, particularly census information. Rural activities, scattered homes in rural areas and lack of correct addresses will impair the validity of the study [13]. There is also often a lack of detailed cartographic data. It is absolutely essential to involve key people in the country and in the area of interest of the study, developing a true partnership, a real co-operation in the sense of a joint operation. Repeated visits to the area targeted by the survey can be useful, as can pilot studies. One should also know how to use tools such as a global positioning system (GPS) and remote sensing from satellites which may generate useful data from current population maps. This technology has become relatively accessible and can provide a sufficiently detailed up-to-date map of the survey area. Such maps are indispensable in areas where maps do not exist and where there are frequent population movements.

Other methodological difficulties must be taken into account. Geographical limits alone are insufficient to define a population. Population censuses are rare and often not confined to a specific period. There are many causes for populations moving: work, sociopolitical factors or war must be considered over a long period. In LMICs, it is common for people to migrate from villages to cities and special economic zones in search of work. Migration may be permanent or show a seasonal pattern. When people come into cities as day labour because agricultural activities are in abeyance, these people often end up living in urban settlements, but retain their original social organization and cultural customs which may be distinctly different from the surrounding population. The stable infrastructure of the city may not necessarily be able to provide access to them for required services (www.unfpa. org/6billion/populationissues/migration.htm). It is important to consider such communities in an epidemiologic survey as they are at higher risk for severe health outcomes - although identifying and following them is a particular challenge.

\section{Census}

When a census has not previously been carried out, it can be done either before or during the main study. Two strategies can be adopted. One can either carry out a door-to-door survey of an entire community or village or 
use a sampling approach. Amongst the first such door-todoor screening protocols for neurological diseases were designed in the 1980s by the World Health Organization [16] and the National Institutes of Health [17]. This protocol was used to carry out community-based surveys in China, Nigeria and India [18-20].

Alternatively, a specific sampling mode can be applied, such as a cluster sampling technique. It does not require prior enumeration of each person, but is based on a list of 'clusters'. In many LMICs this approach makes possible the estimation of the burden of epilepsy more rapidly in a given setting at a much lower cost. Most frequently, villages are taken as clusters [21]. They are selected by lot to have all their inhabitants included in the survey. Thus, cluster surveys provide a geographical grouping of villages on which to focus the investigation. However, it is a challenge to be certain that all the inhabitants of a village have been included. Also, cluster surveys require higher sample sizes than conventional surveys as the analysis must take the clusters into account. WHO simulations indicated that double the study population is required. Cluster sampling can also be used in urban areas, using for example districts or parts of the town as clusters, but this practice could lead to biases since the clusters do not necessarily represent correctly the whole population since people could be diversely dispersed in the town according to their ethnicity, dialect, or whether or not they live in urban slums. Other more complex sampling methods may be used [22]. The awareness of local authorities and the local population is even more important, and the protocol should include procedures which fully explain the course of events.

\section{Screening}

Identification of cases may also cause methodological challenges. Some disorders carry an important stigma. Epilepsy may be seen as associated with possession, or as a contagious disease [23]. This can result in the concealment of cases, even during door-to-door interviews, which remain the gold-standard neuroepidemiological approach in LMICs. Normally, concealing epilepsy is more difficult in rural areas than in the city. Another possibility is that people with focal or absence seizures may not realize that their problem is actually epilepsy, particularly if they have more traditional views of the illness.

In many studies, the head of the household is targeted and is interviewed on behalf of the entire household. A study in Mexico found that interviewing heads of households was inadequate and considerably underestimated the prevalence of epilepsy [24]. Multiple sources of infor- mation may be useful to complement door-to-door surveys as was shown in the Benin survey [25]. Information obtained door-to-door was supplemented with medical data from hospital records, clinics, pharmacies, etc. and by non-medical information from key informants, village chiefs, traditional healers and teachers. Using multiple sources of information allows the application of the capture-recapture method which is a statistical method that is used to calculate corrected prevalence rates taking into account information from three sources: the head of the household, medical data and non-medical information [25].

Rescreening a sample of a population will also help identify PWE missed in the initial survey. False-negative rates can be calculated to give a 'maximum estimated prevalence' [26].

The same epidemiological assessment tools and definitions should be used throughout, despite diverse ethnicity, the existence of many different dialects, perceptions and socio-cultural backgrounds. Hence, interviewers and interpreters where necessary need substantial training to ensure that the questions used can be understood by the different population segments and responded to in the same manner. In addition, quality control measures must be put in place to ensure the consistency of the procedures throughout the data collection period. Questionnaires should be clearly adapted, validated, translated into the dialects used, and retranslated back into the language of origin to check their comprehensibility $[17,20,27,28]$. In LMICs the screening questionnaire is designed to identify convulsive seizures only. Hence, other kinds of epilepsy are omitted in most such studies. The inclusion of questions regarding non-convulsive seizures increases sensitivity, but decreases specificity of the questionnaire, overburdening the few available study neurologists. It is particularly important that questionnaires are pretested for specificity and sensitivity in the same community prior to carrying out the pilot survey. To avoid prejudicing the actual sample population, the testing of the questionnaire should not be done with people actually residing in the area to be surveyed, nor should the pilot study be carried out among people to be included in the main study [17]. To test the questionnaire, it is necessary to have an adequate sample of people with and without epilepsy. Such testing is usually carried out in the setting of a hospital or clinic. However, it is difficult to ensure that the community on whom the questionnaire is being tested is similar to that in a distant rural area.
Bharucha/Odermatt/Preux 


\section{New Technologies}

Today, new technologies offer tremendous opportunities to facilitate data collection, manage and analyse it, and communicate results. Electronic data collection is about to replace paper-based surveys. It has several advantages. Devices such as mobile phones, using short message service (SMS), have effectively been used in LMICs in collecting data and communicating findings in disease control programs [29]. However, sending large, complex amounts of data by SMS is difficult. Personal data assistants (PDAs) allow the collection of more complex data. The development of Android (Google Inc.) platform applications has led to so-called 'smart' devices (primarily touch-screen mobile devices, such as smart phones and tablet computers) with a multitude of applications. Many devices offer the additional advantage of having built-in GPS which automatically captures geographic co-ordinates from external GPS devices. The earlier process of transcribing co-ordinates to paper from external GPS devices is circumvented, thus minimizing transcription errors in the field. A recent comparison of the smart device versus the paper-based survey showed that the smart device collected data of similar quality at a much lower cost [30]. Therefore, an evaluation of PDA devices in surveys of PWE would be most welcome. The perception of PWE to these devices should be taken into consideration.

\section{Diagnosis}

Methodological difficulties may also relate to diagnosis which is mainly clinical. An eye-witness account is essential. A cell phone can be used by the eye witness to record the seizure on video. Patients identified positive on the screening questionnaire should be seen by a clinician as soon as possible. This is often difficult for logistic reasons. Also, ideally, the clinician should be a neurologist with experience in epilepsy as it is important to make a correct diagnosis and to document seizure semiology in order to rule out non-epileptic events and to distinguish between focal and generalized seizures. Specialists trained in neurology or epidemiology are scarce, in particular in LMICs where they would be particularly important.

Electroencephalography (EEG) is necessary to identify absence seizures and to define a specific syndrome. There is often a shortage of EEG equipment and, more importantly, of appropriately trained people to perform and interpret an EEG in the field. Video EEG monitoring is useful for electrophysiological and clinical co-relation, but is rarely available and affordable. Both computed tomogra-

Methodological Difficulties in the Conduct of Neuroepidemiological Studies in LMICs phy scans and magnetic resonance imaging are rarely available facilities and require substantial resources of health providers and patients. If available, they are frequently undergoing maintenance or have broken down. Lack of clinical data, EEG and imaging data results in difficulty in classifying seizures and epilepsy.

\section{Definitions}

The diagnosis of epilepsy should be made using uniform guidelines. A 1993 International League Against Epilepsy (ILAE) commission required at least 2 unprovoked seizures more than $24 \mathrm{~h}$ apart for a definition of epilepsy. Febrile seizures, acute symptomatic seizures, status epilepticus and multiple seizures within $24 \mathrm{~h}$ were thereby excluded [31]. Furthermore, without neuroimaging it is impossible to exclude certain kinds of acute symptomatic seizures such as those due to a dying neurocysticercal granuloma. Hospital-based studies suggest that neurocysticercosis is a major cause of seizures and epilepsy in developing countries [32]. It is important to distinguish between acute symptomatic seizures caused by an active neurocysticercal lesion and unprovoked seizures due to a resolved brain lesion. Most patients who have recovered from neurocysticercosis do not develop epilepsy later [33]. Correspondingly, those studies which fail to exclude patients who have had acute symptomatic seizures due to neurocysticercosis show more epilepsy due to neurocysticercosis than studies which have excluded these patients [34]. A 2005 definition of epilepsy by ILAE and the International Bureau of Epilepsy (IBE) stated that epilepsy is a cerebral disorder manifesting with a single seizure and having an 'enduring predisposition to generate epileptic seizures' and with 'neurobiologic, cognitive, psychological and social consequences' [35]. While this definition might come closer to the true nature of the disorder, it is much harder to translate it into a practical tool to be applied in studies in settings where predominantly health workers with little training are involved in diagnosing, reporting and treating patients. A further problem is the new and different ILAE classification of epilepsy by aetiology. The terms idiopathic, symptomatic and cryptogenic have been replaced by genetic, structural/metabolic and unknown categories $[36,37]$. Changing definitions of epilepsy causes problems in comparing results both within and among settings and countries.

It is also important to distinguish between lifetime epilepsy and active epilepsy. Unfortunately, active epilepsy has been defined in different ways - using a cut-off of the last seizure occurring 1, 2 or 5 years prior to identifica- 
tion $[37,38]$. One reason given for using a shorter duration is that people may have problems recalling past seizures. Differing definitions of active epilepsy lead to different estimates of burden of epilepsy and treatment gap $[6,32]$.

\section{Determining the Age of Study Participants}

An additional problem in some remote rural areas of LMICs is the difficulty in determining the precise age of subjects, particularly if they are older. Indeed, official papers are often missing and some may be falsified for various reasons. The knowledge of age is required to provide age-specific/age-adjusted rates. Without such data, it is not possible to accurately compare rates between the usually young and older populations of LMICs. Different methods of age estimation have been developed and implemented, including the use of historical events to avoid such difficulties [39]. Age of onset of epilepsy is also inadequately recalled and even sometimes concealed by married women who did not disclose their epilepsy at the time of marriage.

Age of onset of epilepsy is an important element to classifying seizures and exploring aetiology. Without knowledge of age at first seizure, duration in years between first and second seizure and age at diagnosis, it is difficult to identify incident cases. Case control and outcome studies should be carried out on incident rather than prevalent cases. If prevalent cases are used, it is not clear whether putative risk factors precede or follow onset of epilepsy. Also, prevalent cases represent a population of those who survive and continue to have seizures, thus resulting in an incidence-prevalence discrepancy. This gap represents those who have had epilepsy but who have died or whose seizures have remitted. The incidence-prevalence gap is much higher in Latin America and Africa than in Asia and developed countries [14, 40, 41]. There are few studies of the natural history of epilepsy in LMICs [42]. Such studies are needed to assess prognosis, i.e. seizure remission and mortality among PWE. Long-term outcome studies of PWE are difficult because people are lost to follow-up. The effect of treatment is difficult to assess because compliance is uncertain. Mbuba et al. [12] found that the sensitivity and the specificity of self-reported adherence was poor. The lack of death certificate data and autopsies make cause of death difficult to ascertain and verbal autopsies have to be relied upon. A review of epilepsy and mortality in Africa found one study which reported a 6-fold increase in mortality in PWE [43] and the cause of death was largely epilepsy related, unlike the situation in high-income countries where epilepsy-related deaths are uncommon. More outcome studies are imperative, particularly using incidence rather than prevalence cohorts.

\section{Socioeconomic Status}

As socioeconomic status is known to be associated with the risk and consequences of epilepsy, it is useful to have a uniformly applicable measure of it $[44,45]$. This would facilitate comparisons of risks and outcomes between populations. In LMICs such as India, socioeconomic status can be considered in two major categories: (i) background status and (ii) current status [Dr. V. Patel, pers. commun.].

\section{Background Socioeconomic Status}

In LMICs such as India, educational status and an index of assets held are easier and more reliable to assess than income. Unlike the situation in high-income countries, income is difficult to assess due to the fact that the vast majority of people work in the unorganized or informal sector. Also, in LMICs, there is an unwillingness to divulge personal information to strangers. In LMICs where basic education is more uniformly available, income becomes a more important criterion. The background socioeconomic status probably influences the risk of epilepsy, for example through inequitable access to quality maternal and child healthcare.

\section{Current Socioeconomic Status}

Current status is measured in LMICs by factors which reflect recent economic distress, such as indebtedness and hunger, and is probably a better index of the consequences of epilepsy. The distinction between background and current socioeconomic status is not as clear as it appears, since assets may be sold to meet current needs.

Both these measures need to be uniformly assessed, using standardized and validated methods. In a recent review of the relationship between poverty and mental health in LMICs, it was found that only $7 \%$ of indicators of poverty were measured by standardized validated methods [46]. Asset-based assessment of socioeconomic status is widely used in researching neglected tropical diseases but less commonly in epilepsy [12]. Its advantage is in allowing comparisons across large areas.

\section{Sociocultural Factors}

Unlike the situation with socioeconomic status, which can be related to both cause and effect of epilepsy, sociocultural factors mainly influence the prognosis and outcome of epilepsy. Beliefs and perceptions influence help-
Bharucha/Odermatt/Preux 
seeking in several ways, including recognition of a seizure disorder, the possibility that medical treatment exists, and the type of treatment sought. Projects aimed both at research into these areas and at training staff would be useful. These should preferably be multidisciplinary and involve both the humanities and social sciences.

\section{Other Problems}

\section{Logistics}

The logistics of providing consumables and medical equipment cause problems. These include transport across insecure regions and severe climatic conditions which make maintaining equipment difficult.

\section{Motivation}

In LMICs, lack of interest in neuroepidemiological studies leads to insufficient knowledge of the extent and impact of epilepsy and hence low priority at all levels of policy making and public health. Many decision-making bodies encounter some difficulty in deciding on resource allocation [47]. At all levels of government - national, regional, district and communal - it is necessary to obtain agreement on those surveys that have been conducted. Ideally, there should be an integrated plan to improve knowledge of neurological epidemiology, which should then be incorporated into the national health programmes.

\section{Funding}

Providing immediate and long-term funding is a major drawback. It is important that requests for funding for high-quality projects be submitted to foundations, which can be appropriate sponsors. Internationally provided money usually targets tuberculosis, malaria and HIV. Other tropical diseases are beginning to receive increasing attention. These are the neglected tropical diseases which have been inadequately addressed in LMICs. They might have a considerable connection with epilepsy or other neurological disorders. It is imperative to organize a real curative service to the screened population in parallel with epidemiological investigations.

\section{Ethics}

Ethical questions are extremely sensitive. There are still far too few independent ethics committees in resource-poor countries. Where no committee exists, obviously health authorities should act, but they are less inde- pendent than a designated body. In addition, signed informed consent is often too complicated to obtain, and it is necessary to establish simple consent protocols that the population can truly understand. Written and signed consent may be impossible because of illiteracy, but written evidence of comprehension and oral consent can be obtained via a witness. The risk/benefit ratio should be accurately evaluated. Finally, all the difficulties related to the appreciation of neuroepidemiological work in tropical countries should not be overlooked even if, overall, they diminish over time.

\section{Quality of Research}

These methodological and logistical difficulties result in the implementation of studies of relatively modest quality. Samples may be small, leading to a lack of power. The accuracy of diagnosis may be lower as equipment is not available. Hence, studies are often published in local journals, which are often not indexed in major international databases. In the major international journals, it is sometimes more difficult to publish a study from an LMIC than similar work from the developed world. However, it is still quite possible if the study is truly original and adequately targeted and if the quality of the translation into English (if, of course, the journal is in English) has been verified by a professional translator. It is best to avoid multiple publications using the same data from a single study.

\section{Conclusion}

Many specific methodological difficulties face those conducting neuroepidemiological studies in LMICs, most of them tropical. Nevertheless, the concepts and methods applied must be the same as those used globally, even if it is clear that their translation into practice may differ. Neuroepidemiological research in these countries is difficult, but feasible, and extremely useful to provide decision-makers with the knowledge they need to allocate limited resources in the best way possible in order to reduce the treatment gap. Genuine co-operation at all levels is absolutely necessary if such research is to be conducted under appropriate conditions. The survey protocol should be particularly detailed and thorough. Specific epidemiological methodologies may be useful, and the collection of data must be standardized. Neuroepidemiological research therefore requires careful organization and 
the development of solutions specific to LMICs. Currently, $90 \%$ of health research addresses $10 \%$ of the world's health problems of the global population. This mismatch should not continue. Increased emphasis on neuroepidemiological studies in LMICs and systematic reviews and meta-analyses of such studies have a role to play in reestablishing a balance.

\section{Acknowledgements}

The authors wish to thank Dr. Roberta H. Raven for her help in the preparation of the manuscript and Mrs. Apoline Fernandes for typing and assistance in collating the references.

\section{Disclosure Statement}

The authors have nothing to disclose.

\section{References}

1 WHO: Atlas: epilepsy care in the world 2005. Geneva, World Health Organization, 2005.

$\checkmark 2$ Meinardi H, Scott RA, Reis R, et al: The treatment gap in epilepsy: the current situation and ways forward. Epilepsia 2001;42:136149.

- 3 Greenhalgh T: How to read a paper: papers that summarise other papers (systematic reviews and meta-analyses). BMJ 1997;315:672.

4 Ngugi AK, Kariuki SM, Bottomley C, et al: Incidence of epilepsy: a systematic review and meta-analysis. Neurology 2011:77: 10051012.

5 Ngugi AK, Bottomley C, Kleinschmidt I, et al: Estimation of the burden of active and lifetime epilepsy: a meta-analytic approach. Epilepsia 2010;51:883-890.

6 Mbuba CK, Ngugi AK, Newton CR, et al: The epilepsy treatment gap in developing countries: a systematic review of the magnitude, causes, and intervention strategies. Epilepsia 2008;49:1491-1503.

7 Meyer AC, Dua T, Ma J, et al: Global disparities in the epilepsy treatment gap: a systematic review. Bull World Health Organ 2010: 88:260-266.

8 Eysenck H: Problems with meta-analysis; in Chalmers I, Altman DG (eds): Systematic Reviews. London, BMJ, 1995.

9 Thompson SG: Why sources of heterogeneity in meta-analysis should be investigated; in Chalmers I, Altman DG (eds): Systematic Reviews. London, BMJ, 1995.

10 Ngugi AK, Bottomley C, Kleinschmidt I, et al: Prevalence of active convulsive epilepsy in Sub-Saharan Africa and associated risk factors: cross-sectional and case-control studies. Lancet Neurol 2013;12:253-263.

11 Crepin S, Houinato D, Nawana B, et al: Link between epilepsy and malnutrition in a rural area of Benin. Epilepsia 2007;48:1926-1933.

$\checkmark 12$ Mbuba CK, Ngugi AK, Fegan G, et al: Risk factors associated with the epilepsy treatment gap in Kilifi, Kenya: a cross-sectional study. Lancet Neurol 2012;11:688-696.

13 Quet F, Odermatt P, Preux PM: Challenges of epidemiological research on epilepsy in resource-poor countries. Neuroepidemiology 2008;30:3-5.
14 Preux PM, Druet-Cabanac M: Epidemiology and aetiology of epilepsy in sub-Saharan Africa. Lancet Neurol 2005:4:21-31.

15 Tran DS, Odermatt P, Le TO, et al: Prevalence of epilepsy in a rural district of central Lao PDR. Neuroepidemiology 2006;26:199-206.

16 WHO Neurosciences Programme: Research protocol for measuring the prevalence of neurological disorders in developing countries. Geneva, World Health Organization, 1982.

17 Schoenberg BS: Clinical neuroepidemiology in developing countries: neurology with few neurologists. Neuroepidemiology 1982; 1: 137-142.

18 Li SC, Schoenberg BS, Wang CC, et al: Epidemiology of epilepsy in urban areas of the People's Republic of China. Epilepsia 1985:26: 391-394.

19 Osuntokun BO, Schoenberg BS, Nottidge VA, et al: Research protocol for measuring the prevalence of neurologic disorders in developing countries: results of a pilot study in $\mathrm{Ni}$ geria. Neuroepidemiology 1982;1:143-153.

20 Bharucha NE, Bharucha EP, Dastur HS: Pilot survey of the prevalence of neurologic disorder in the Parsi community of Bombay. Am J Prev Med 1987;3:293-299.

21 Preux PM, Chea K, Chamroeun H, et al: Firstever, door-to-door cross-sectional representative study in Prey Veng province (Cambodia). Epilepsia 2011:52:1382-1387.

22 Houinato D, Preux PM, Charriere B, et al: Interest of LQAS method in a survey of HTLVI infection in Benin (West Africa). J Clin Epidemiol 2002;55:192-196.

23 Rafael F, Houinato D, Nubukpo P, et al: Sociocultural and psychological features of perceived stigma reported by people with epilepsy in Benin. Epilepsia 2010;51:1061-1068.

24 Quet F, Preux PM, Huerta M, et al: Determining the burden of neurological disorders in populations living in tropical areas: who would be questioned? Lessons from a Mexican rural community. Neuroepidemiology 2011;36:194-203.

25 Debrock C, Preux PM, Houinato D, et al: Estimation of the prevalence of epilepsy in the Benin region of Zinvie using the capture-recapture method. Int J Epidemiol 2000;29: 330-335.
26 Placencia M, Sander JWAS, Shorvon SD, et al: Validation of a screening questionnaire for the detection of epileptic seizures in epidemiological studies. Brain 1992;115:783-794.

27 Preux PM: Questionnaire in a study of epilepsy in tropical countries (in French). Bull Soc Pathol Exot 2000;93:276-278.

28 Diagana M, Preux PM, Tuilla M, et al: Screening for epilepsy in tropical areas: validation of a questionnaire in Mauritania (in French). Bull Soc Pathol Exot 2006;99:103-107.

29 Déglise C, Suggs LS, Odermatt P: SMS for disease control in developing countries: a systematic review of mobile health applications. J Telemed Telecare 2012;18:273-281.

30 King JD, Buolamwini J, Cromwell EA, Panfel A, Teferi T, Zerihun M, Melak B, Watson J, Tadesse Z, Vienneau D, Ngondi J, Utzinger J, Odermatt P, Emerson PM: A novel electronic data collection system for large-scale surveys of neglected tropical diseases. PLoS One 2013; 8:e74570.

-31 Commission on Epidemiology and Prognosis, International League against Epilepsy: Guidelines for epidemiologic studies on epilepsy. Epilepsia 1993;34:592-596.

32 Carpio A, Hauser WA: Epilepsy in the developing world. Curr Neurol Neurosci Rep 2009; 9:319-326.

33 Singh G, Singh P, Singh I, et al: Epidemiologic classification of seizures associated with neurocysticercosis: observations from a sample of seizure disorders in neurologic care in India. Acta Neurol Scand 2006;113:233-240.

34 Carpio A, Hauser WA: Remote symptomatic epilepsies and epileptic syndromes: etiological spectrum; in Murthy JMK, Senanayake N (eds): Epilepsy in the Tropics. Austin, Landes Bioscience, 2006.

35 Fisher RS, van Emde Boas W, Blume W, et al: Epileptic seizures and epilepsy: definitions proposed by the International League Against Epilepsy (ILAE) and the International Bureau for Epilepsy (IBE). Epilepsia 2005;46:470472 
36 Berg AT, Berkovic SF, Brodie MJ, et al: Revised terminology and concepts for organization of seizures and epilepsies: report of the ILAE Commission on Classification and Terminology, 2005-2009. Epilepsia 2010;51: 676-685.

37 Thurman DJ, Beghi E, Begley CE, et al: Standards for epidemiologic studies and surveillance of epilepsy. Epilepsia 2011;52(suppl 7):2-26.

38 Shorvon SD, Farmer PJ: Epilepsy in developing countries: a review of epidemiological, sociocultural, and treatment aspects. Epilepsia 1988;29(suppl 1):S36-S54.
39 Paraiso MN, Houinato D, Guerchet M, et al: Validation of the use of historical events to estimate the age of subjects aged 65 years and over in Cotonou (Benin). Neuroepidemiology 2010:35:12-16.

40 Burneo JG, Tellez-Zenteno J, Wiebe S: Understanding the burden of epilepsy in Latin America: a systematic review of its prevalence and incidence. Epilepsy Res 2005;66:63-74.

41 Mac TL, Tran D-S, Quet F, et al: Epidemiology, aetiology, and clinical management of epilepsy in Asia: a systematic review. Lancet Neurol 2007;6:533-543.

42 Bharucha NE, Shorvon SD: Epidemiology in developing countries; in Engel J Jr, Pedley TA (eds): Epilepsy: A Comprehensive Textbook. Philadelphia, Lippincott-Raven Publishers, 1997, pp 105-118.

43 Diop AG, Hesdorffer DC, Logroscino G, Hauser WA: Epilepsy and mortality in Africa: a review of the literature. Epilepsia 2005; 46(suppl 11):33-35.
44 Heaney DC, Beagley CE: Economic evaluation of epilepsy treatment: a review of the literature. Epilepsia 2002;43(suppl 4):10-16.

45 Birbeck G, Chomba E, Atadzhanov M, et al: The social and economic impact of epilepsy in Zambia: a cross-sectional study. Lancet Neurol 2007;6:39-44.

46 Cooper SC, Lund C, Kakuma R: The measurement of poverty in psychiatric epidemiology in LMIC: critical review and recommendations. Soc Psychiatry Psychiatr Epidemiol 2012;47:1499-1516.

47 Dumas M, Preux PM: Epilepsy in tropical areas (in French). Bull Acad Natl Med 2008;192: 949-960. 